\title{
Submitted: The ultrasonographic dynamic heel-rise test Accepted: of the Achilles tendon
} 02.06.2021

Published: 16.08.2021

\author{
Beata Ciszkowska-Łysoń1,2, Urszula Zdanowicz ${ }^{3,4}$, Robert Śmigielski ${ }^{5}$ \\ ${ }^{1}$ LIFE Institute's Orthopaedics Department and Biological Treatment Centre, LIFE Institute, \\ Warsaw, Poland \\ ${ }^{2}$ Department of Radiology, Carolina Medical Center, Warsaw, Poland \\ ${ }^{3}$ Orthopaedic and Sports Traumatology Department, Carolina Medical Center, Warsaw, Poland \\ ${ }^{4}$ McGowan Institute for Regenerative Medicine, University of Pittsburgh, USA \\ ${ }^{5}$ LIFE Institute's Orthopaedics Department and Biological Treatment Centre, LIFE Institute, \\ Warsaw, Poland \\ Correspondence: Beata Ciszkowska-Łyson, LIFE Institute's Orthopaedics Department and \\ Biological Treatment Centre, LIFE Institute, Grzybowska 43a, 00-855 Warsaw, Poland; \\ e-mail:bciszkowska@lifeinstitute.pl
}

DOI: $10.15557 / J o U .2021 .0043$

\section{Keywords}

Achilles tendon rupture,

Achilles tendon ultrasonography, heel-rise test, Achilles tendon reconstruction, Achilles tendon function assessment

\begin{abstract}
The treatment of Achilles tendon rupture attempts to restore the primary anatomical structure and principal biomechanical properties of the damaged tendon. Postoperative clinical assessment of the healing progression and function monitoring may be difficult and require experience. Diagnostic imaging (ultrasonography and magnetic resonance imaging) helps monitor the healing process. In the following paper, we propose a heel-rise test - a dynamic assessment of the Achilles tendon performed under direct observation and ultrasound monitoring to establish the tension of the Achilles tendon. The test allows for a simple assessment of tendon function and may be safely repeated at any postoperative stage. It may be performed by a physician, radiologist and physiotherapist to monitor the recovery process following Achilles tendon damage.
\end{abstract}

\section{Motto}

The aim of Achilles tendon surgery is not to simply suture it, but to create conditions allowing to fully restore its function.

\section{Introduction}

The Achilles tendon (AT) is made of tendinous bands arising from the heads of the triceps surae muscle attaching to the calcaneal tuberosity. Despite being the largest and strongest tendon in the human body, the AT can become ruptured. The injury often occurs in physically active individuals who expect to resume full sports activity after treatment. The treatment of a ruptured AT attempts to restore its primary anatomical structure and principal biomechanical properties. To ensure optimal healing and restore the length of the tendon, various surgical techniques to connect or bring closer the torn tendon stumps are used, including open repair and percutaneous repair.

The patient's foot alignment and Thompson squeeze tests as well as Achilles tendon resting angle (ATRA) are considered the testing modalities designed to assess the normal resting function of the AT. Based on these tests, a traumatic tendon rupture or secondary rupture can be suspected quite clearly. Yet still, postoperative clinical assessment of the recovery progress may be difficult and require an appropriate level of experience. Moreover, it may be further impeded by the formation of adhesions in the tissues surrounding the healing tendon, which often makes the clinical evaluation of the tendon difficult. Diagnostic imaging plays a supportive role in the clinical assessment of the patient. It allows to monitor the recovery in terms of the tendon's structure status as well as biomechanical properties through dynamic evaluation. Radiological imaging provides information about two major aspects of the patient's progress: tendon morphology following the reconstruction, and healing process assessment pointing to possible issues occurring throughout the postoperative treatment protocol, including physical therapy. The overall condition of 


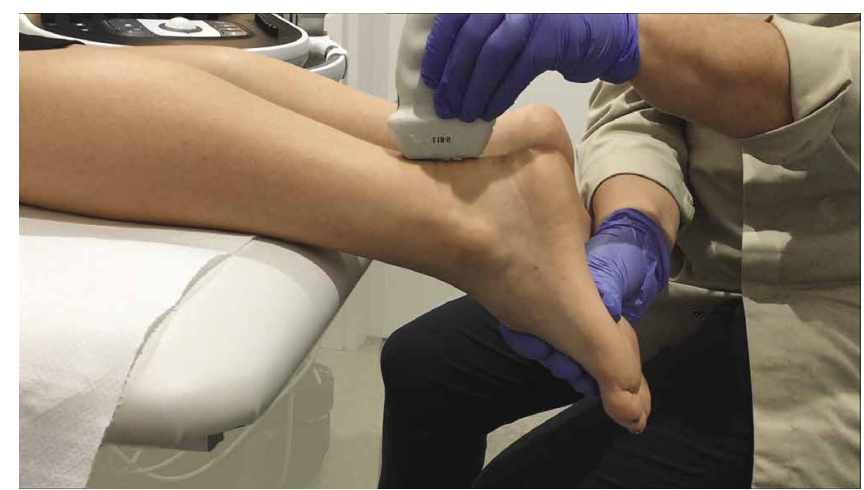

Fig. 1. Technique: the initial stage of the dynamic assessment. The transducer is applied longitudinally above the calcaneal tuberosity, along the AT, allowing for direct evaluation of the presence of the tendon fibres' resting tension, indicating a good function of the muscle-tendon unit, or blurred tendon structure with no fibrillar structure, which may indicate an impaired healing process

a particular patient and the characteristics of the tendon healing process should also be taken into account.

The diagnostic imaging modalities used to assess the healing process of a torn AT include ultrasonography (US) and magnetic resonance imaging (MRI). Both methods are suitable for evaluating the condition of the tendon's healing structure following injury. In addition, US offers the advantage of a dynamic assessment of the tendon.
The development of the US heel-rise test and its implementation into the ultrasound AT assessment protocol stems from observations made during orthopaedic clinical examinations done routinely at our Clinic. During the examination, the orthopaedist asks the patient to flex the ankle actively against the resistance from their hand and then evaluates the tension of the AT through palpation. In some patients, we observed that despite bending the ankle with the maximum force achievable by the patient, one could feel with the fingers that the AT was loose over the upper outline of the calcaneus. In such cases, after applying the US transducer, we observed that the AT was not tight, but wavy. This observation gave the authors the idea to perform the test on a regular basis.

This, in turn, means that during the US heel-rise test the patient flexes the ankle actively using the strength of the other flexor muscles instead of the triceps calf muscle and the AT.

This report aims to present a complementary dynamic ultrasound AT evaluation method that employs an element of the clinical examination protocol (dynamic heel-rise test), albeit with direct ultrasound monitoring of tendon activity.

\section{Protocol for the dynamic ultrasound-guided heel-rise test}

Dynamic US test is performed to assess AT traction - the transfer of the force of the triceps surae muscle contraction to the distal tendon. The dynamic test enables the
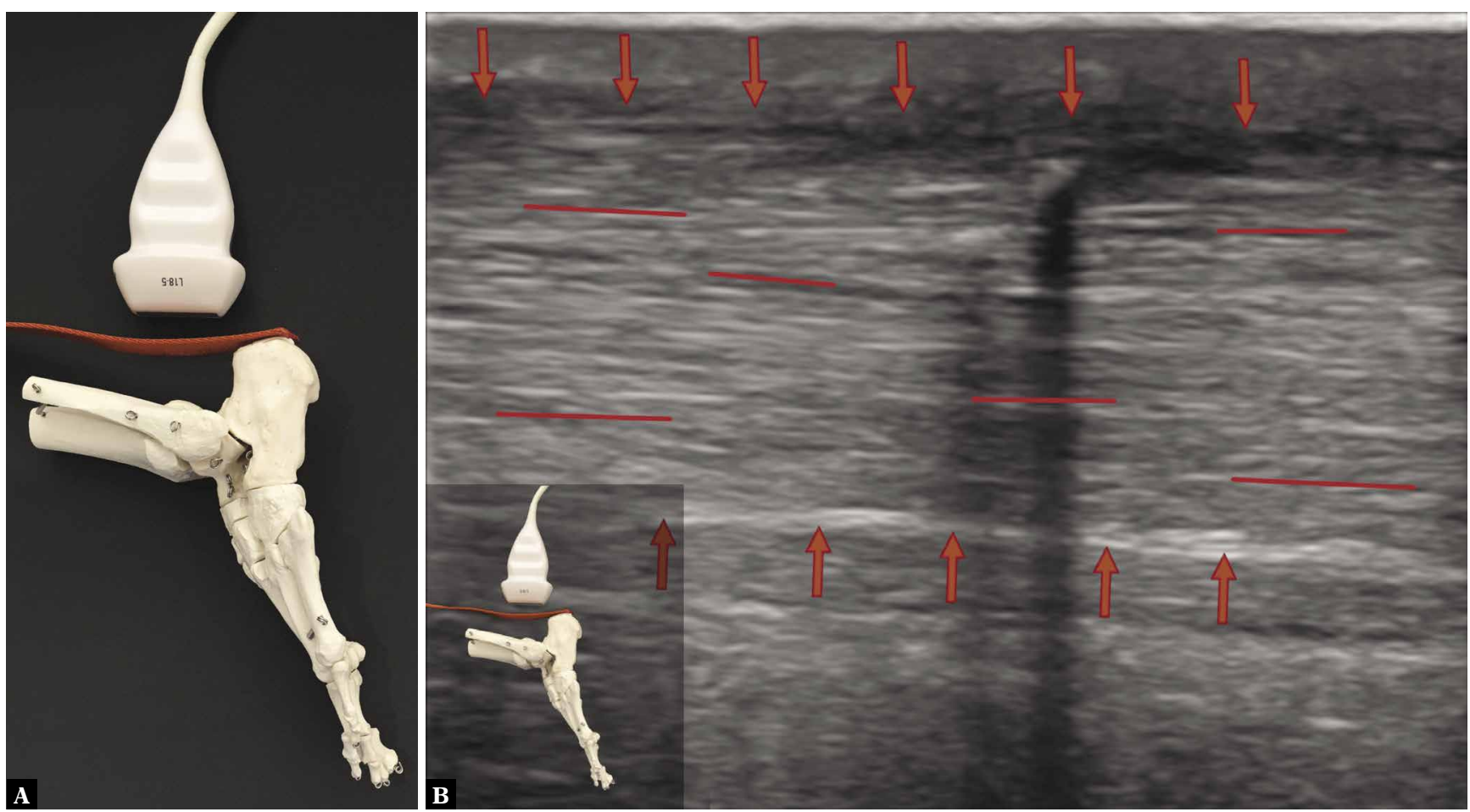

Fig. 2. Ultrasound examination protocol. A. The transducer is applied along the AT, above the calcaneal tuberosity. The tendon tension is present at rest. B. An 18-5 MHz. US image of a tendon at post-rupture remodelling phase (4.5 months postoperatively). A longitudinal plane, a linear arrangement of AT fibres [red short lines]; the image indicates the presence of resting tension. [red arrows mark the borders of the tendon] 

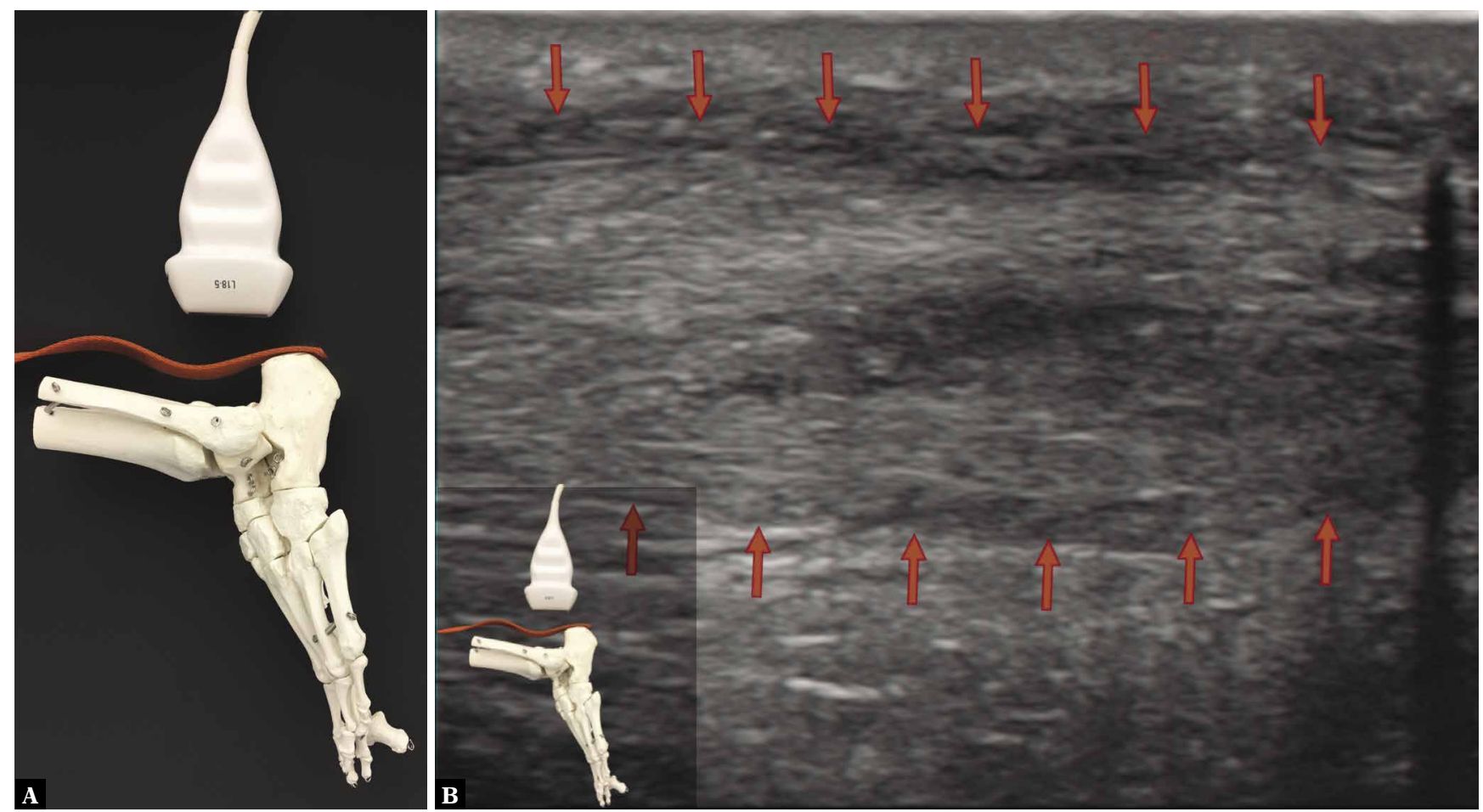

Fig. 3. Ultrasound examination protocol. A. The transducer is applied along the AT, above the calcaneal tuberosity. At rest, the tendon is undulated, there is no tension in the AT, which is visible on ultrasound as blurred tendon structure with no linear fibrillar arrangement of the fibres. An abnormal image. B. An 18-5 MHz. US image of a tendon during the remodelling phase at 3 months after the surgery. Longitudinal plane, there is no linear arrangement of AT fibres; blurred tendon structure, hypoechoic zones are present within the tendon; the image indicates the absence of resting tension. [red arrows mark the borders of the tendon]

diagnostician to observe the presence of AT tension during resisted active plantar flexion of the ankle joint. Such an evaluation allows to identify potential tendon undulation ${ }^{(1,2)}$ due to the lack of tendon activity and its passiveness during foot movement executed by other plantar flexors. This additional test, while included in the ultrasound examination protocol, offers a fast and reliable way to determine the tension of the AT and may be performed during any ultrasound appointment.

\section{Technique}

A routine postoperative protocol for AT patients at our Clinic includes regular follow-up orthopaedic appointments combined with ultrasound examinations including the US heel-rise test at 3 and 6 weeks, 3, 6, and 9 months, as well as 1 year after AT surgery.

The ultrasound-guided assessment of the biomechanical behaviour of a reconstructed tendon is performed in two positions: at rest and during active ankle plantar flexion against resistance.

Stage one: the patient is in a prone position with both feet at rest, placed beyond the edge of the examination table to allow for unrestricted foot movement. A linear transducer is applied longitudinally to the AT above the calcaneal tuberosity to obtain the best possible image of the longitudinally arranged tendon fibres (Fig. 1).
If resting tension is present in the muscle-tendon unit, a fibrillar structure of the AT should be evident in the image (Fig. 2). The absence of a fibrillar structure in the tendon indicates the lack of resting tension in the muscle-tendon unit (Fig. 3).

Stage two: the patient is in a prone position with both feet at rest, placed beyond the edge of the examination table. The linear transducer is again applied longitudinally to the AT, above the upper outline of the calcaneal tuberosity. The transducer is placed so that the image of longitudinally arranged tendon fibres during tendon movement can be observed. The patient is asked to perform a maximum pain-free plantar flexion of the ankle. Fig. 4 shows the hand of the diagnostician placed on the metatarsus, providing resistance against the foot during active plantar flexion, and thus forcing active muscle tension in the lower leg, including the triceps surae muscle and consequently the AT, similarly to the heel-rise test.

Our experience indicates that active plantar flexion of the ankle joint with the patient in a prone position is safe if performed carefully, even in the early postoperative period - (1 week). The resistance applied against the foot may be gradually increased, with the major indicator of the maximum strength allowed being the pain threshold of each individual patient. This means that, over time, the applicable resistance would increase from low to high at the more advanced phases of tendon healing which occur at 6 months up to 1 year after 


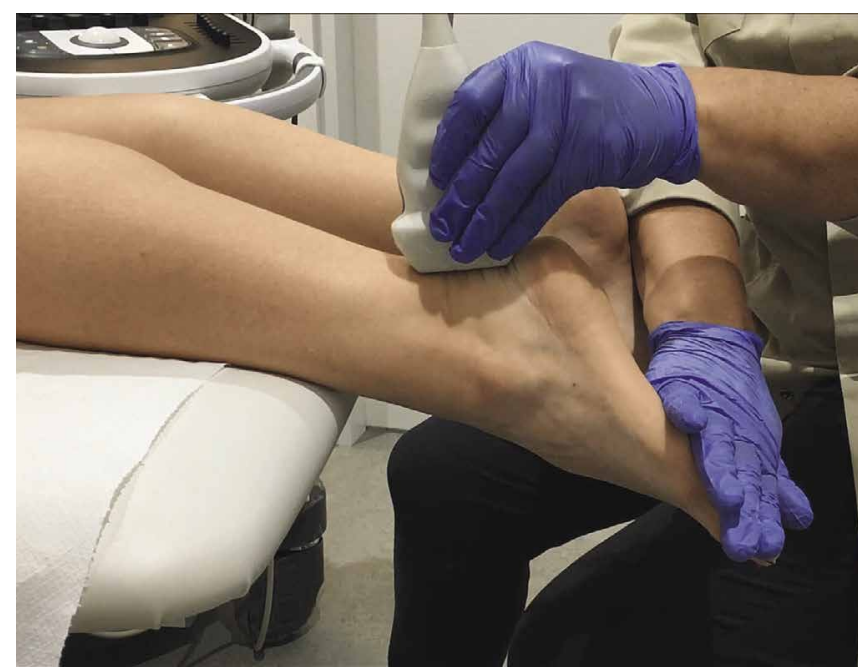

Fig. 4. Technique: a US heel-rise test - the plantar flexion of the ankle with resistance applied against the foot imitating the classical heel-rise test; maximum active plantar flexion of the ankle, the diagnostician's hand applies ground-like resistance to the foot, thereby forcing tension in the calf muscles. The transducer is placed longitudinally above the calcaneal tuberosity, along the Achilles tendon, which allows direct observation of the moving tendon. An ultrasound examination allows to directly assess the presence of tension of tendon fibres during active movement, indicating good function or tendon fibre undulation pointing to elongation during healing. In the case of elongation of a healing tendon, the ankle joint is not bent by the force of the triceps surae muscle, but by the strength of the other plantar flexors
Achilles tendon surgery(3). The examiner's hand resists pressure from the patient's foot. The presence of tensed fibres of the AT should be monitored on the ultrasound during active foot movement against resistance (Fig. 5). Tendon fibre undulation could be identified on the ultrasound images throughout the entire movement, or in some cases, only at the end-stage of plantar flexion of the ankle. In both scenarios, the lack of tension of the AT suggests its elongation during postoperative period (Fig. 6). In the case of AT elongation, the ankle joint does not bend as a result of the triceps surae muscle activity but through the employment of the other plantar flexors, hence no AT tension is observed.

\section{Comments}

The presence of both resting and active tension of the AT is indicative of good condition of the muscle-tendon unit both in terms of anatomy and structure ${ }^{(4)}$. The presence of tendon tension during motion in a closed kinematic chain is a sign of restoration of good tendon function and is of particular importance for the clinical assessment (Fig. 7).

Elongation of the AT scar after suturing a completely ruptured tendon is believed to be the cause of treatment failure. Studies have shown that surgical restoration of the length of a ruptured tendon prevents the loss of strength in the calf triceps muscle ${ }^{(5)}$.
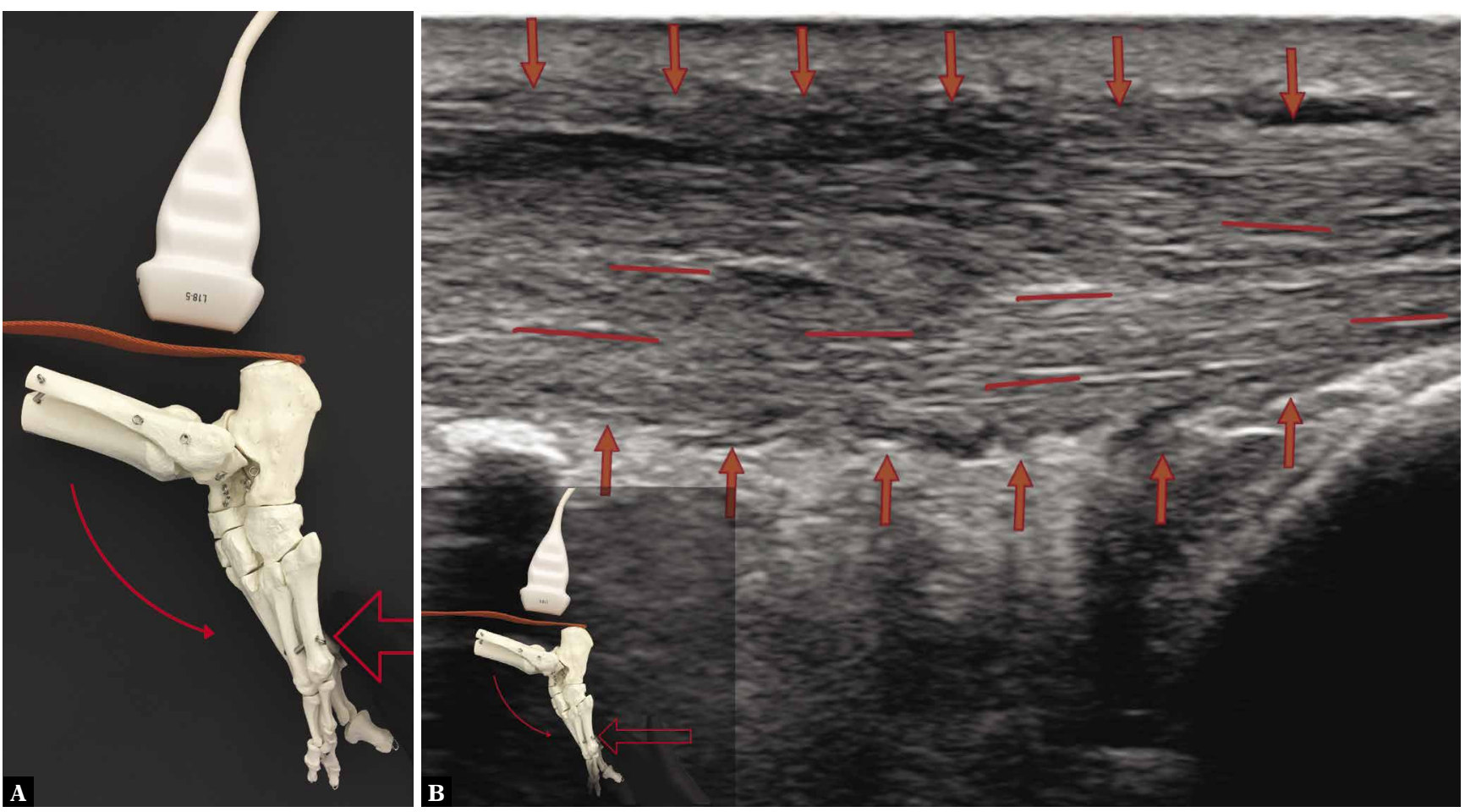

Fig. 5. Ultrasound examination protocol. A. The transducer is placed longitudinally above the calcaneal tuberosity, along the AT; active plantar flexion [curved arrow] of the ankle joint with the diagnostician's hand applying ground-like foot resistance [straight arrow] as in the classical heel-rise test flexion. Evident tension of the AT. The heel-rise movement is made by the force of the triceps surae muscle with the tension of the AT. The image indicates a good function of a surgically treated AT. B. A longitudinal 18-5 MHz US image of the AT; evident fibrillar structure of the tendon fibres [red short lines]; good tendon tension; the image indicates a good function of the AT. [red arrows mark the borders of the tendon] 

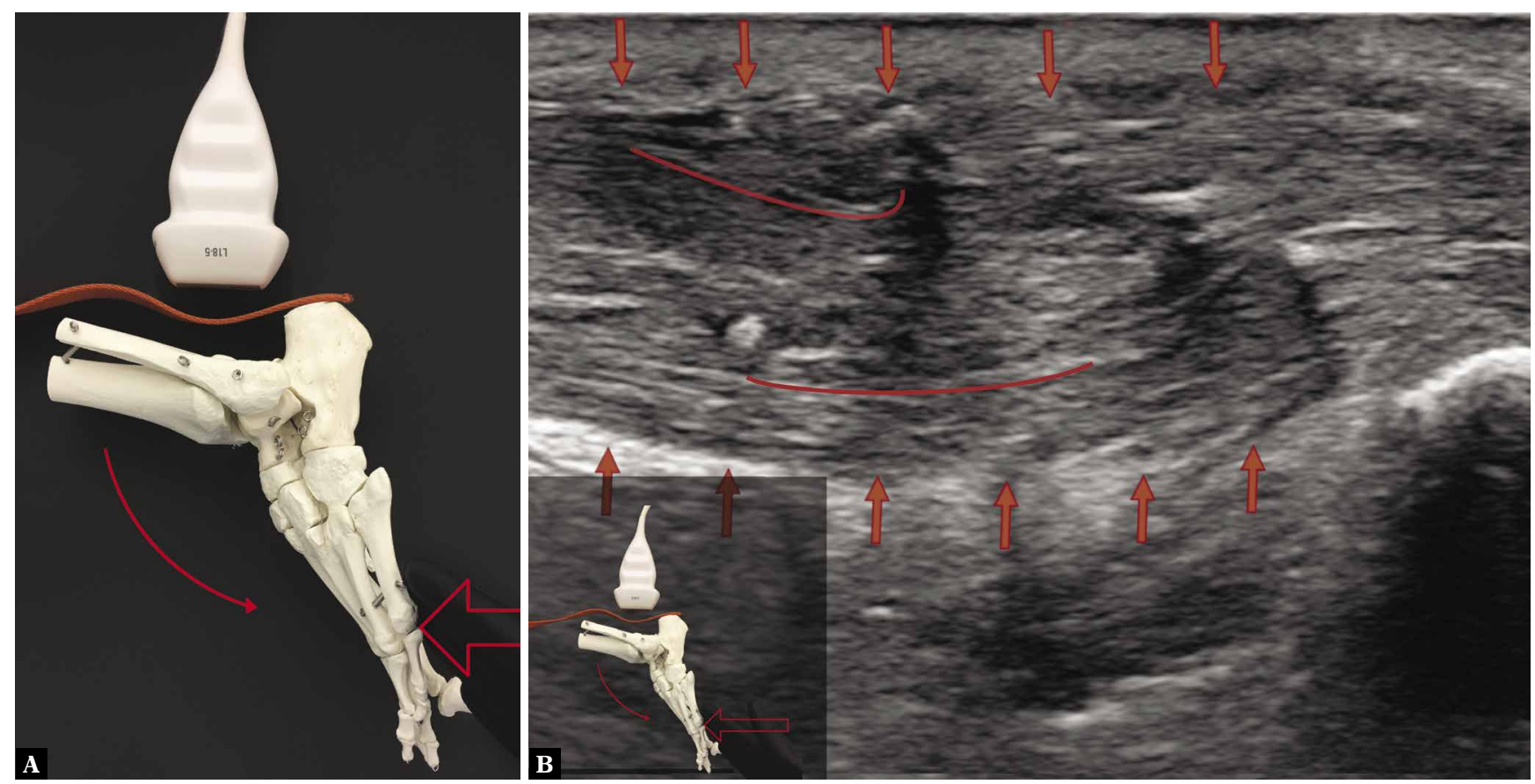

Fig. 6. Ultrasound examination protocol. A. The transducer is placed longitudinally above the calcaneal tuberosity, along the AT; active maximum plantar flexion of the ankle joint [curved arrow] with the diagnostician's hand applying ground-like foot resistance [straight arrow] as in the heel-rise test. No tension is seen in the AT, which is undulated. The heel-rise movement is performed not by the force of the triceps surae muscle, but with the strength of the other plantar flexors. The image indicates a dysfunction of the surgically treated AT, healing with elongation of the tendon. B. A longitudinal 18-5 MHz US image of the AT, the evident fibrillar structure, but the tendon fibres appear undulated in the dynamic imaging [the curved red lines indicate the wavy course of the fibres], indicating the lack of tension; the tendon is loose [red arrows mark the borders of the tendon]
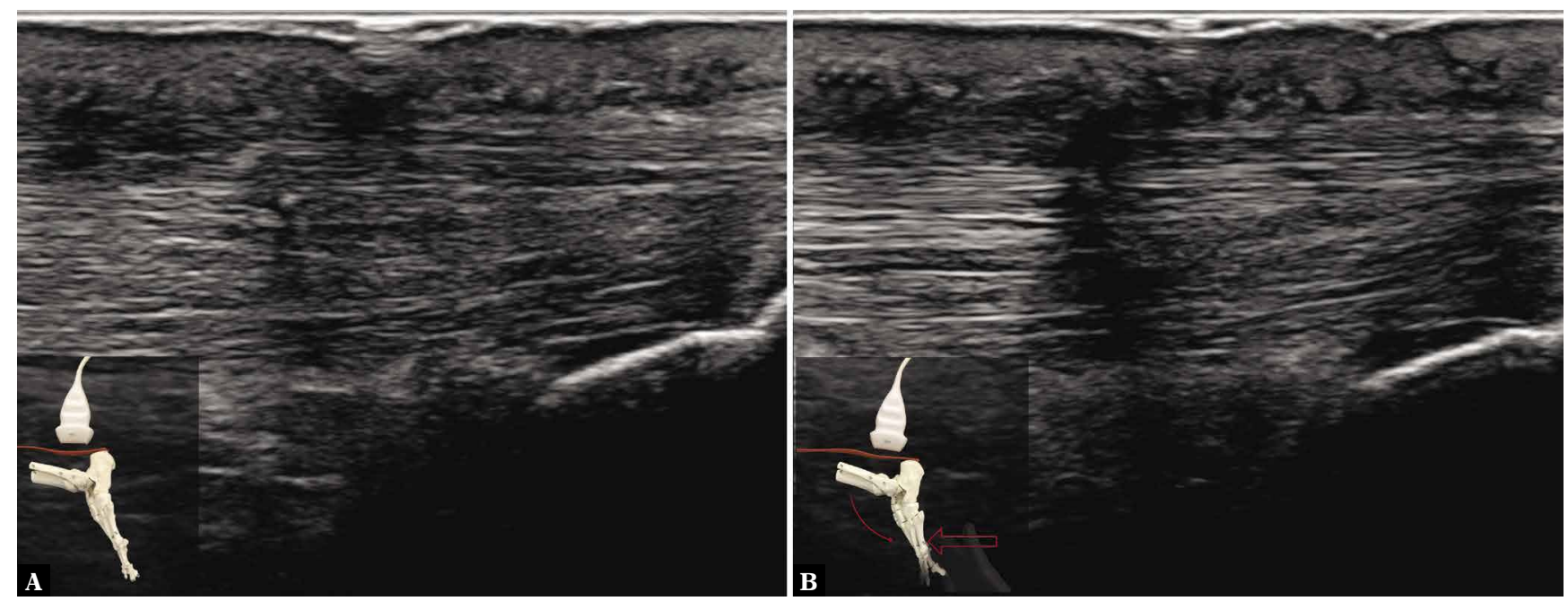

Fig. 7. The US heel-rise test, an examination 3 months after AT rupture reconstruction - the longitudinal 18-5 MHz US images of the AT; on the left (A) a fibrillar structure of the tendon fibres is visible - the image indicates the presence of resting tension; on the right (B) - during active plantar flexion of the ankle with applied resistance, the fibres of the AT are linear, no waving of the fibres is observed. The image indicates a good function of the AT

A specific task of the AT is to maintain tension during the final phase of plantar flexion - for example during jumping and sprinting. Therefore, evaluating this feature is essential for monitoring the tendon function. Elongation of the AT by about $2-3$ centimetres after the treatment of tendon rupture, although seemingly insignificant, disturbs the function of plantar flexion of the ankle joint. This, in turn, results in permanent impairment of the ankle joint biomechanics. The resulting loss of proper function of the muscles of the lower leg and the knee escalates further during walking, running and jumping, i.e. basic sports activities ${ }^{(6,7)}$.

The lack of tension may result from tendon elongation, inadequate activation of the calf triceps muscle, adhesions 

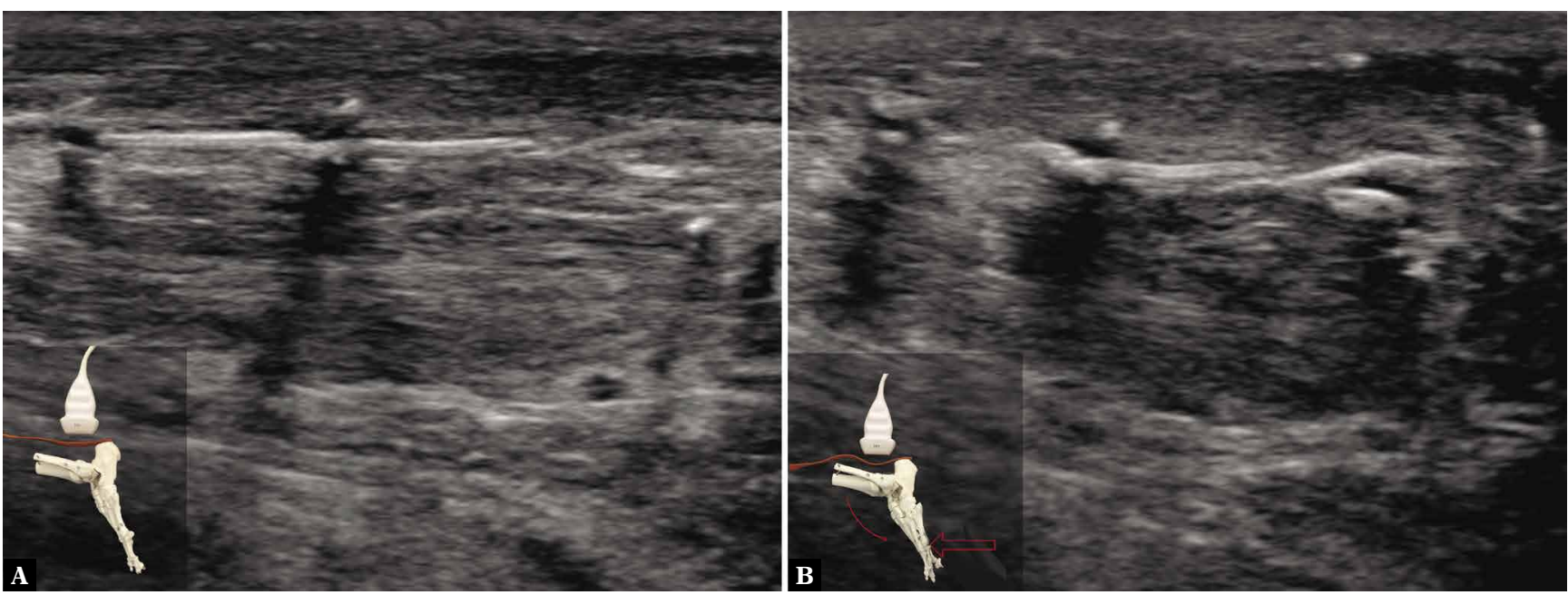

Fig. 8. The US heel-rise test, an examination 6 weeks after AT rupture reconstruction - the longitudinal 18-5 MHz US images of the AT; on the left (A) the fibrous structure of the tendon is not clearly visible, but there is no waviness of the tendon-the image indicates the presence of resting tension of AT; on the right $(\mathbf{B})$ - during active plantar flexion of the ankle with resistance, wavy tendon fibres, and wavy surgical sutures are visible - indicating the lack of AT tension; the tendon is loose
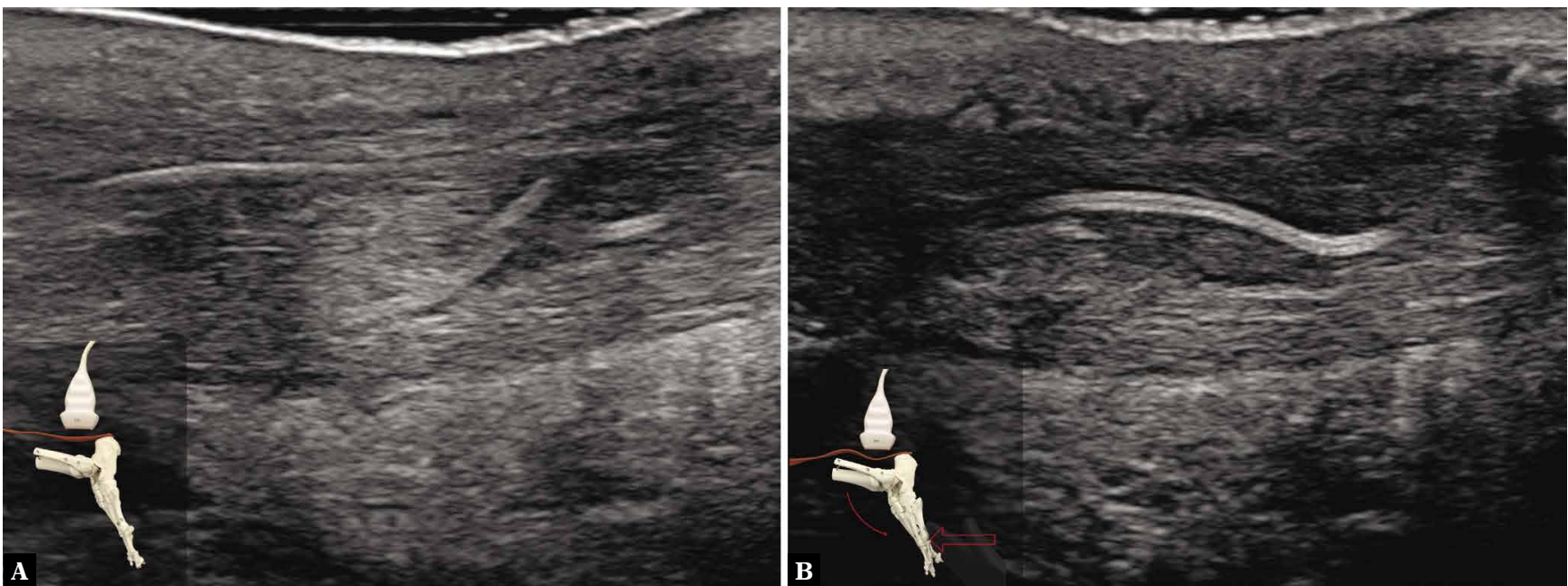

Fig. 9. The US heel-rise test, an examination 3 months after AT rupture reconstruction - the longitudinal 18-5 MHz. US images of the AT; one the left (A) the fibrous structure of the tendon is not clearly visible, but there is no waviness of the tendon - the image does not show the resting tension of the tendon; on the right $(\mathbf{B})$ - during active plantar flexion of the ankle with applied resistance, wavy tendon fibres, and wavy surgical sutures are visible - indicating the lack of AT tension; the tendon is loose

around the muscles and tendon, or a combination thereof. The presence of undulations, i.e. the lack of tension in the $A T^{(2,8)}$, especially in the final phase of plantar flexion (Fig. 8 and Fig. 9), indicates that the movement is not performed with the force of the triceps surae muscle, but with the strength of plantar flexors, i.e. a heel rise is possible, but without the involvement of the triceps surae muscle/AT ${ }^{(9)}$.

Improper AT healing with its elongation correlates with reduced strength of plantar flexion assessed by biomechanical testing ${ }^{(8)}$ and abnormal heel-rise test. Interestingly, biomechanical impairment may also develop in the other limb, most likely as a result of adjustment to the limitations of the affected limb ${ }^{(9)}$.

In case of a classical heel-rise test, reduced calf muscle strength in the final phase of plantar flexion is caused by elongation of the $\mathrm{AT}^{(5,8)}$, which results in a reduced number of heel-rise test repetitions, lower heel-rise height or inability to perform the test. The benefit of the US heel-rise test is that it may be performed in cases where the classical test is not yet possible. This is a significant advantage, as the test can be performed at 6 weeks up to 6 months after the injury when the tendon scar lengthens the most ${ }^{(10,11)}$, and information on the tendon healing process is crucial. The US heel-rise test can be frequently repeated and is particularly clinically useful when healing disorders occur.

The US heel-rise test is cost-effective and accessible, and moreover it does not require any additional ultrasound equipment. An ultrasound device with a linear transducer is sufficient. The results are assessed qualitatively for the presence or absence of AT tension during plantar flexion. 
The US heel-rise test is dedicated to monitoring the function of the Achilles tendon regardless of the surgical technique used or in the evaluation of conservative treatment. The test is not meant to measure the length of the tendon. It only shows the tendon tension or lack thereof. The surgical techniques were not discussed because, regardless of the treatment, the ultimate goal is to regain the function of the AT. The lack of tension on the AT equals unsatisfactory AT function.

Insufficient AT function may have a complex aetiology which is not the subject of this brief report. Of course, in the daily practice, a full clinical and radiological evaluation of the patient is considered, especially in cases of treatment failure.

\section{Conclusion}

The ultrasound heel-rise test is a simple yet significant addition to the Achilles tendon function assessment protocol. It brings together the elements of both clinical and ultrasound examinations.

The question for the orthopaedic surgeons as well as diagnosticians is whether the US heel-rise test is something they need in their everyday practice, and if it can aid their evaluation process and help optimise the treatment.

The authors believe that the test has value for several reasons. It provides a "second", independent opinion concerning the structural condition, but more importantly,

\section{References}

1. Sasaki K, Yamamoto N, Kiyosawa T, Sekido M: The role of collagen arrangement change during tendon healing demonstrated by scanning electron microscopy. J Electron Microsc (Tokyo) 2012; 61: 327-334.

2. Franchi M, Fini M, Quaranta M, De Pasquale V, Raspanti M, Giavaresi G et al.: Crimp morphology in relaxed and stretched rat Achilles tendon. J Anat 2007; 210: 1-7.

3. Sharma P, Maffulli N: Biology of tendon injury: healing, modeling and remodeling. J Musculoskelet Neuronal Interact 2006; 6: 181-190.

4. Jamali AA, Afshar P, Abrams RA, Lieber RL: Skeletal muscle response to tenotomy. Muscle Nerve 2000; 23: 851-862.

5. Mullaney MJ, McHugh MP, Tyler TF, Nicholas SJ, Lee SJ: Weakness in end-range plantar flexion after achilles tendon repair. Am J Sports Med 2006; 34: 1120-1125.

6. Brorsson A, Willy RW, Tranberg R, Grävare Silbernagel K: Heel-rise height deficit 1 year after achilles tendon rupture relates to changes in ankle biomechanics 6 years after injury. Am J Sports Med 2017; 45: 3060-3068. the function of the healing tendon. Such input gives the surgeon a significant argument for or, as the case may be, against allowing the patient to extend the set of permitted activities. Secondly, the test can be performed by the orthopaedist during the appointment, thus offering a complementary tool that could be used during the clinical examination, especially when adhesions surrounding the AT make palpation difficult. Additionally, the test can be done by a physiotherapist as a form of biofeedback allowing to optimise the physical therapy protocol.

All in all, the test offers considerable benefits at little to no cost.

\section{Acknowledgments}

The study was part of Novel Scaffold-based Tissue Engineering Approaches to Healing and Regeneration of Tendons and Ligaments (START) project, co-funded by The National Centre for Research and Development (Poland) within STRATEGMED programme (STRATEGMED1/233224/10/NCBR/2014).

\section{Conflict of interest}

The authors do not report any financial or personal connections with other persons or organizations, which might negatively affect the contents of this publication and/or claim authorship rights to this publication.
7. Willy RW, Brorsson A, Powell HC, Willson JD, Tranberg R, Silbernagel KG: Elevated knee joint kinetics and reduced ankle kinetics are present during jogging and hopping after achilles tendon ruptures. Am J Sports Med 2017; 45: 1124-1133.

8. Carmont MR, Zellers JA, Brorsson A, Nilsson-Helander K, Karlsson J, Silbernagel KG: Age and tightness of repair are predictors of heel-rise height after achilles tendon rupture. Orthop J Sport Med 2020; 8: 2325967.

9. Finni T, Hodgson JA, Lai AM, Edgerton VR, Sinha S: Muscle synergism during isometric plantarflexion in achilles tendon rupture patients and in normal subjects revealed by velocity-encoded cine phase-contrast MRI. Clin Biomech 2006; 21: 67-74.

10. Silbernagel KG, Steele R, Manal K: Deficits in heel-rise height and Achilles tendon elongation occur in patients recovering from an Achilles tendon rupture. Am J Sports Med 2012; 40: 1564-1571.

11. Diniz P, Pacheco J, Guerra-Pinto F, Pereira H, Ferreira FC, Kerkhoffs G: Achilles tendon elongation after acute rupture: is it a problem? A systematic review. Knee Surg Sports Traumatol Arthrosc 2020; 28: 4011-4030. 